\title{
On the Resource Allocation Problem in Wireless Networked Control Systems
}

\author{
Themistoklis Charalambous, Ayca Ozcelikkale, Mario Zanon, Paolo Falcone and Henk Wymeersch
}

\begin{abstract}
This paper considers the scheduling problem of a decentralized system where a number of dynamical subsystems with no computational power are scheduled to transmit their measurements via a resource-limited communication network to a remote decision maker who acts as an estimator, controller and scheduler for the subsystems. We propose a new approach for communication resource allocation for a wide class of objective functions, for both coupled and decoupled systems, and for both scheduling observations as well as control commands. This framework allows to schedule over a finite horizon and can explicitly deal with stochastic channels. For decoupled subsystems, we propose the notion of cost of information loss (CoIL) and we demonstrate that the communications resource allocation problem can be directly expressed in terms of CoIL functions as an assignment-type optimization problem. Illustrative examples demonstrate how communication resources affect the performance of the system.
\end{abstract}

\section{INTRODUCTION}

Control and estimation of dynamical systems under communication constraints have been investigated under a wide range of scenarios (see [1], [2] for early developments). Optimal control for independent and identically distributed (i.i.d.) packet dropping links have been studied in [2], [3]. A stochastic sensor scheduling strategy for a dynamical system is developed and upper and lower bounds on the expected error covariance are provided in [4]. Convex relaxations for sensor selection problem for state estimation with a finite look-ahead window are proposed and sublinear performance loss of using shorter windows is investigated in [5]. Sensor scheduling for minimization of steady-state estimation error and stabilization for a single dynamical system for both reliable and packet-dropping links is considered in [6]. Sensor scheduling for i.i.d. packet dropping links are considered and optimality of threshold policies for the single sensor case is shown [7].

Periodic sensor scheduling solutions are recognized as an important class of optimal solutions for varying problem settings [8]-[11]. Optimal periodic sensor scheduling solutions for a two-sensor scenario are derived in [8]. It is shown that schedules with bounded average estimation error can be approximated by bounded periodic schedules for infinite horizon scheduling problems [9], [10]. The estimation error, the boundedness of the schedules and the trajectory that infinite-horizon schedules converge to for are shown to be independent of the initial covariance matrices [9], [10].

A. Ozcelikkale, M. Zanon, P. Falcone and H. Wymeersch are with the Department of Signals and Systems, Chalmers University of Technology, Göteborg, Sweden. e-mail: \{name.surname@chalmers.se

T. Charalambous is with the Department of Electrical Engineering and Automation, School of Electrical Engineering, Aalto University, Espoo, Finland. e-mail: \{name.surnamedaalto.fi\}
Similar results for sensing of multiple dynamical systems over an infinite horizon are obtained in [11].Formulations using a value function associated with measurements of a sensor have been considered in the context of control of dynamical systems [12]-[16] and hypothesis testing [17]. For minimization of both the final or average estimation error, myopic policies, policies which look ahead only one time-step, are shown to be optimal through the usage of a generalized information gain function under some regularity conditions [13]. By using an importance indicator based on the difference between the mean-square error estimate at local sensors and the prediction at the central processor side, sensor scheduling for a shared channel is investigated in [16]

In this work, we consider such communication resource allocation schemes under a unified framework by explicitly incorporating control performance in our performance metric. Inspired by the works of [12], [14], we analyze our setup for the LQG scenario, but unlike their approach, our work does not use current measurements. The communications resources are limited and as a result, which agents are allowed to transmit and which communication resource is allocated to each agent has to be carefully designed by a remote decision maker. To quantify the "value" of the measurements of each sensor, we propose and utilize the concept of "Cost of Information Loss" (CoIL), which is the opposite of the Value of Information (VoI) used in other papers (see, e.g., [14], [18]). This cost is based on the statistical properties of the sensor measurements. We consider both the scenario where the communication is guaranteed to be successfully completed when a channel is allocated to a specific channel, such as in CAN-like network scenarios for communications [14], and the more general scenario where packet losses may occur during transmission [2]. The channel seen by each agent has different statistical properties, hence each agent has a different probability of success on each channel. We discuss the scenarios with both coupled and decoupled subsystems through their states and we show how the communications resource allocation problem can be directly expressed as an assignment-type optimization problem.

The rest of the paper is organized as follows. Section II describes the setup of the networked control system we consider and Section III suggests a framework for analyzing the performance of the system. Subsequently, in Sections V and VI, we use the LQG framework as an example for decoupled and coupled systems, respectively. The performance of a networked control system for various scenarios is demonstrated via simulations in Section VII. Finally, in Section VIII draw conclusions and discuss directions for future work. 


\section{SyStem Model AND PRELIMINARIES}

\section{A. Notation}

The sets of real numbers are denoted by $\mathbb{R}$. Vectors are denoted by small letters, matrices are denoted by capital letters, and sets by calligraphic capital letters. The transpose of matrix $A$ is denoted by $A^{T} . \mathbb{E}\{\cdot\}$ represents the expectation of its argument. The transpose of a matrix or vector $A$ is denoted by $A^{T}$. For a square matrix $A \in \mathbb{R}^{n \times n}$, we denote by $A \succ 0$ the positive-definite matrix $\mathrm{A}$, and by $\operatorname{diag}\{A\}$ the matrix having entries on its diagonal and zero elsewhere. $\operatorname{tr}\{\cdot\}$ denotes the trace and $\operatorname{cov}(\cdot)$ denotes the covariance of a matrix. The cardinality of a set $\mathcal{A}$ is denoted by $|\mathcal{A}|$.

\section{B. Agents and central components}

We consider a system consisting of: (a) a set $\mathcal{N}$ of agents (dynamical subsystems) with no computational power, $|\mathcal{N}| \geq$ 1 , that obtains noisy information about the state of the agent and possibly other agents; and (b) a remote decision maker who acts as a central estimator, a central controller, and a central resource allocator for the agents. Based on a resource allocator, some of the sensors transmit their measurements to a remote decision maker who acts as an estimator, control designer and scheduler for the agents; see Fig. 1. Each agent possibly has different dynamical and statistical properties and, hence, the effect of the loss of a sensor measurement for the overall system performance varies.

The agents are characterized by a system of equations

$$
x_{k+1}=h\left(x_{k}, u_{k}, w_{k}\right),
$$

where $x_{k} \in \mathbb{R}^{n_{x}}$ is the state of the system, $n_{x}=\sum_{i=1}^{N} n_{x_{i}}$, and $n_{x_{i}}$ is the number of states of agent $i$ at time $k ; u_{k} \in \mathbb{R}^{n_{u}}$ is the control input, $n_{u}=\sum_{i=1}^{N} n_{u_{i}}$, and $n_{u_{i}}$ is the number of inputs of agent $i ; w_{k} \in \mathbb{R}^{n_{x}}$ is a stochastic disturbance with zero mean and finite second order matrix $W$, and $h$ : $\mathbb{R}^{n_{x}} \times \mathbb{R}^{n_{u}} \times \mathbb{R}^{n_{x}} \mapsto \mathbb{R}^{n_{x}}$ is a function, whose properties ensure that there is a solution for system (1). System (1) is the interconnection of $\mathrm{N}$ agents and it can be decomposed to

$$
x_{i, k+1}=h_{i}\left(x_{i, k}, u_{i, k}, w_{i, k}\right)+\sum_{j \in\left|\mathcal{N}_{i}^{\text {in }}\right|} g_{i j}\left(x_{j, k}\right),
$$

where $x_{i, k} \in \mathbb{R}^{n_{x_{i}}}$ is the state of agent $i$ at time $k$, $u_{i, k} \in \mathbb{R}^{n_{u_{i}}}$ is the control input, $w_{i, k} \in \mathbb{R}^{n_{x_{i}}}$ is a stochastic disturbance with zero mean and finite second order matrix $W_{i}$, with functions $h_{i}: \mathbb{R}^{n_{x_{i}}} \times \mathbb{R}^{n_{u_{i}}} \times \mathbb{R}^{n_{x_{i}}} \mapsto \mathbb{R}^{n_{x_{i}}}$ and $g_{i j}: \mathbb{R}^{n_{x_{j}}} \mapsto \mathbb{R}^{n_{x_{i}}}$. All agents that link to node $i$ directly are said to be in-neighbors of agent $i$ and belong to the set $\mathcal{N}_{i}^{\text {in }}$. If the agents are not interconnected $g_{i j}=0$ and (2) reduces to

$$
x_{i, k+1}=h_{i}\left(x_{i, k}, u_{i, k}, w_{i, k}\right) \text {. }
$$

It is also assumed that agents have noisy (possibly partial) observations $y_{i, k} \in \mathbb{R}^{n_{y_{i}}}$ of the state of the system $x_{k}$, i.e.,

$$
y_{i, k}=\ell_{i}\left(x_{k}, v_{i, k}\right),
$$

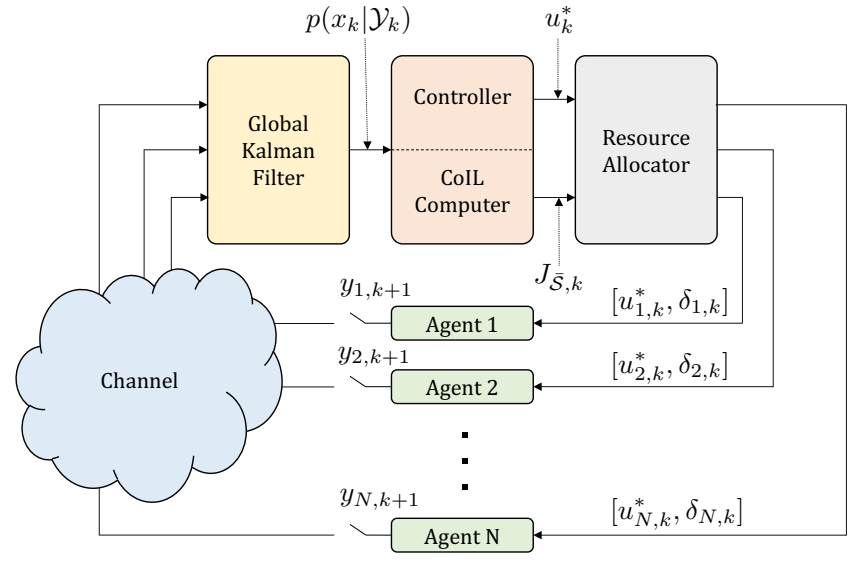

Fig. 1. System architecture: agents transmit their measurements to the estimator, provided the resource allocator provides them a slot. The resource allocator decides on the allocation of the communication resources, given the information from the controller and cost of information loss (CoIL).

where $v_{i, k} \in \mathbb{R}^{n_{y_{i}}}$ is a stochastic disturbance with zero mean and finite second order matrix $V$, independent of $w_{i, k}$, and $n_{y_{i}}$ is the number of observations, and function $\ell_{i}: \mathbb{R}^{n_{x}} \times \mathbb{R}^{n_{y_{i}}} \mapsto \mathbb{R}^{n_{y_{i}}}$. Agent $i$ has the possibility to send its observations $y_{i, k} \in \mathbb{R}^{n_{y_{i}}}$ to the central estimator over uplink channels.

The estimator keeps track of the distribution $p\left(x_{k} \mid \mathcal{Y}_{k}\right)$, where $\mathcal{Y}_{k}=\cup_{i} \mathcal{Y}_{i, k}$ is the set of all observations from all agents up to time $k$ and $\mathcal{Y}_{i, k}$ is the set of observations sent by agent $i$ sent to the estimator up to time $k$.

The controller takes as input $p\left(x_{k} \mid \mathcal{Y}_{k}\right)$, and computes the control command $u_{k}^{*} \triangleq\left[\begin{array}{lll}u_{1, k} & \ldots & u_{N, k}\end{array}\right]$, which is to be sent to the agents over downlink channels. The controller performs the following computation

$u_{k}^{*}=\arg \min _{u} \mathbb{E}_{x}\{f(u \mid x)\}=\arg \min _{u} \int p\left(x_{k} \mid \mathcal{Y}_{k}\right) f(u \mid x) d x$,

where $f(u \mid x)$ represents the cost of control action $u$, given that the system is in state $x$. The cost function subsumes all constraints in the objective.

The resource allocator assigns communication resources (time slots, power, bits, etc.) to: (i) the downlink channels to each agent; (ii) the uplink channel from each agent.

\section{Channel and resources model}

For the resource allocator to assign communication resources in a meaningful way, we must have a model for the communication and the way communication losses impact the controller. The uplink from agent $i$ to the controller is determined by the probability of successful reception, $p\left(r_{i}^{\text {up }}\right) \in[0,1]$, where $r_{i}^{\text {up }}$ represents the uplink communication resources allocated to agent $i$. In this work, the downlink from the resource allocator to agent $i$ is assumed to be ideal, i.e., the probability of successful reception, $p\left(r_{i}^{\mathrm{dn}}\right) \in[0,1]$, of the downlink communication resources, $r_{i}^{\mathrm{dn}}$, is equal to 1. Our objective is to allocate the limited resources so that the system's overall cost is minimized. 


\section{CoIL Framework AND Resource AllocAtor}

We focus on collision-free medium-access control (MAC) protocols where access to a specific channel resource block is only given to only one agent at a given time, such as frequency-division multiple access (FDMA) schemes and time-division multiple access (TDMA) schemes. The effective number of available sub-channels, i.e., resource blocks, that can be used by the agents is smaller than the number of agents. Such scenarios are encountered in resource-limited multiple access schemes when the number of available frequency bands or time-slots are smaller than the number of agents that would like to communicate.

\section{A. Input from controller to resource allocator}

The resource allocator receives the control commands $u_{k}$ from the controller. The resource allocator assigns the communication resources $r^{u p} \subseteq\left[\begin{array}{lll}r_{1}^{\mathrm{up}} & \ldots & r_{N}^{\mathrm{up}}\end{array}\right]$. For such an allocation to be meaningful for the control, we must (i) define the actions taken by the central controller when information is missing, and (ii) quantify the cost of missing information in the uplink.

1) Actions under missing information: The controller can still compute $p\left(x_{i, k} \mid \mathcal{Y}_{k}\right)$ and compute a new control action accordingly.

2) Cost of only missing uplink information: The absence of uplink information means that the controller does not (necessarily) have access to the latest observations. Let $\mathcal{S} \subseteq$ $\mathcal{N}$ denote the set of agents from which the central estimator received their measurements and $\overline{\mathcal{S}}$ the rest of the agents, i.e., $\mathcal{S} \cup \overline{\mathcal{S}}=\mathcal{N}$ and $\mathcal{S} \cap \overline{\mathcal{S}}=\emptyset$. Then, at time step $k+1$,

$$
\begin{array}{ll}
p\left(x_{i, k+1} \mid \mathcal{Y}_{k}, y_{\mathcal{S}, k+1}, u_{k}^{*}\right), & \text { for } i \in \mathcal{S}, \\
p\left(x_{i, k+1} \mid \mathcal{Y}_{k}, \emptyset, u_{k}^{*}\right), & \text { for } i \in \overline{\mathcal{S}},
\end{array}
$$

where we explicitly include the control command $u_{k}^{*}$ already computed at time $k$. Here, $y_{\mathcal{S}, k+1}$ denotes the values of the observations from the agents in the set $\mathcal{S}$ at time $k+1$. Now, to capture the cost of this lost information (CoLI), we define

$$
p_{\text {ref }}\left(x_{k+1}\right)=p\left(x_{k+1} \mid \mathcal{Y}_{k+1}, u_{k}^{*}\right),
$$

i.e., the distribution of the aggregate state at time $k+1$ when no information is lost. We now introduce

$$
\begin{gathered}
J_{\overline{\mathcal{S}}, k}^{\mathrm{up}}=\min _{u} \int p\left(x_{\overline{\mathcal{S}}} \mid \mathcal{Y}_{k}, \emptyset, u_{\overline{\mathcal{S}}, k}^{*}\right) p\left(x_{\mathcal{S}} \mid \mathcal{Y}_{k}, y_{\mathcal{S}, k+1}, u_{\mathcal{S}, k}^{*}\right) \\
f\left(u \mid x_{\mathcal{S}}, x_{\overline{\mathcal{S}}}\right) \mathrm{d} x_{\overline{\mathcal{S}}} \mathrm{d} x_{\mathcal{S}}-\min _{u} \int p_{\text {ref }}(x) f(u \mid x) \mathrm{d} x,
\end{gathered}
$$

where $x_{\mathcal{A}}$ denotes the aggregate state vector for the agents in set $\mathcal{A}$ and $u_{\mathcal{A}, k}^{*}$ denotes the set of optimum controls at time $k$ for the agents in set $\mathcal{A}$, where $\mathcal{A}=\mathcal{S}, \overline{\mathcal{S}}$. Since the observations $y_{\mathcal{S}, k+1}$ are not known at time $k$, we replace them with their most likely value, derived from (2) or (3). In other words, we consider a distribution derived from a synthetic observation for the for the agents for which information will be received and a distribution from the dynamics (2) or (3), when no information will be received. We subtract the cost corresponding to perfect downlink and uplink information, in order for $J_{\overline{\mathcal{S}}, k}^{\mathrm{up}}$ to capture the marginal cost of lost uplink information. We note that $J_{\overline{\mathcal{S}}, k}^{\text {up }} \geq 0$ and $J_{\overline{\mathcal{S}}, k}^{\text {up }} \geq J_{\overline{\mathcal{S}}^{\prime}, k}^{\text {up }}$ for $\overline{\mathcal{S}} \supseteq \overline{\mathcal{S}}^{\prime}$.

\section{B. Resource Allocator}

The aim of the resource allocator is to schedule the available communication resource blocks to agents.

1) Reliable Channels: We first consider the scenario where the effective channels on each resource block are reliable. Hence if an agent is assigned to a resource block, then there is no outage and its message is guaranteed to be received by the remote agent. where $r$ is the number of channels available. Let $\delta_{i, k}$ be an index showing whether the observation of agent $i$ at time $k$ is received by the estimator

$$
\delta_{i, k}= \begin{cases}1, & \text { observation transmitted } \\ 0, & \text { otherwise }\end{cases}
$$

Hence, we consider the following optimization problem

$$
\bar{J}_{k}=\min _{\delta_{k}: \sum_{i} \delta_{i, k} \leq r} J_{\overline{\mathcal{S}}, k}^{\text {up }}
$$

where $\overline{\mathcal{S}}=\left\{i: \delta_{i, k}=0\right\}$ by definition.

2) Unreliable Channels: Here we focus on the case where the channels are possibly unreliable. Hence, even an agent is assigned to a certain channel, its message is not guaranteed to arrive at the remote center. We are interested in optimizing the average performance over channel statistics.

Let $\mathcal{F}$ be the index set of available channels with $|\mathcal{F}|=r$, where $r$ is arbitrary. Let us denote the decision variables $\mathcal{R}=\left\{r_{i, j, k}, \forall i, \forall j\right\}$ with $r_{i, j, k} \in\{0,1\}$ where $r_{i, j, k}$ denotes whether agent $i \in \mathcal{N}$ transmits at channel $j \in \mathcal{N}$ at time step $k$. Here $r_{i, j, k}=0$ indicates that no transmission takes place, and $r_{i, j, k}=1$ indicates that transmission takes place. A channel can be only used by one agent at a given time

$$
\sum_{i \in \mathcal{N}} r_{i, j, k} \leq 1, \quad \forall j, \forall k .
$$

Each agent can only use one channel at a time

$$
\sum_{j \in \mathcal{N}} r_{i, j, k} \leq 1, \quad \forall i, \forall k .
$$

We note that $r_{i, j, k}$ denotes whether there is a communication attempt or not, and due to unreliable channels, neither $r_{i, j, k}$ for any $j$ nor $\sum_{j} r_{i, j, k}$ is not necessarily equal to $\delta_{i, k}$. Instead, we have the following

$$
\begin{aligned}
& \mathbb{P}\left(\delta_{i, k}=1 \mid r_{i, j, k}=1\right)=q_{i, j, k} \\
& \mathbb{P}\left(\delta_{i, k}=0 \mid r_{i, j, k}=1\right)=1-q_{i, j, k}
\end{aligned}
$$

where $q_{i, j, k}$ denote the success probability of agent $i$ on channel $j$ at time $k$ given that there is a communication attempt by agent $i$ on channel $j$. Given $r_{i, j, k}$, success events are statistically independent across all agents, channels and time instants. We are interested in the following optimization

$$
\min _{\mathcal{R}} \mathbb{E}\left\{J_{k} \mid \mathcal{R}\right\}
$$

subject to (8) and (9). Here, the expectation is with respect to (w.r.t.) the channel statistics. 


\section{Resource Allocation}

\section{A. Decoupled Dynamics}

1) Reliable Channels: We now focus on a system in which agents have decoupled, linear dynamics and observations. Hence the optimization problem can be written as

$$
\bar{J}_{k}=\min _{\delta_{k}: \sum_{i} \delta_{i, k} \leq r} \sum_{i \in \mathcal{N}} E_{i, k},
$$

where $\delta_{k} \triangleq\left[\begin{array}{lll}\delta_{1, k} & \ldots & \delta_{N, k}\end{array}\right]$ and $E_{i, k}$ is the cost for agent $i$ at time step $k$. We define $E_{i, k}^{0}$ the cost for agent $i$ when its observation is not received; similarly, $E_{i, k}^{1}$ represents the cost for agent $i$ when its observation is received. Based on the definition of CoIL, the cost function associated with losing measurements from agent $i$ at time step $k$ can be written as

$$
J_{\{i\}, k}=\sum_{l \in \mathcal{N}, l \neq i} E_{l, k}^{1}+E_{i, k}^{0}-\sum_{l \in \mathcal{N}} E_{l, k}^{1}=E_{i, k}^{0}-E_{i, k}^{1} .
$$

Hence, the total cost of the system at time step $k$, denoted by $J_{k}$, can be expressed in terms of $J_{\{i\}, k}$ as follows:

$$
\begin{aligned}
J_{k} & =\sum_{i \in \overline{\mathcal{S}}} E_{i, k}^{0}+\sum_{i \in S} E_{i, k}^{1} \\
& =\sum_{i \in \overline{\mathcal{S}}} E_{i, k}^{0}-\sum_{i \in \overline{\mathcal{S}}} E_{i, k}^{1}+\sum_{i \in \mathcal{N}} E_{i, k}^{1} \\
& =\sum_{i \in \overline{\mathcal{S}}} J_{\{i\}, k}+\sum_{i \in \mathcal{N}} E_{i, k}^{1}
\end{aligned}
$$

Since $\sum_{i \in \mathcal{N}} E_{i, k}^{1}$ does not depend on the set $S$, minimization of $J_{k}$ over $S$ is equivalent to minimization of $J_{k}-\sum_{i \in \mathcal{N}} E_{i, k}^{1}$. Hence, the optimization problem of minimizing the total cost, as generally expressed in (6), can be equivalently written as

$$
\min _{\overline{\mathcal{S}}} \sum_{i \in \overline{\mathcal{S}}} J_{\{i\}, k},
$$

in which $\overline{\mathcal{S}}$ is selected as the set of $|\mathcal{N}|-r$ agents with the smallest $J_{\{i\}, k}$. Equivalently, by selecting $\mathcal{S}$ as the set of $r$ agents with the highest $J_{\{i\}, k}$ as follows

$$
\max _{\mathcal{S}} \sum_{i \in \mathcal{S}} J_{\{i\}, k}
$$

We note that this optimization problem is an assignment problem [19], [20]. Since assignment problems are typically presented with a slightly different notation, for the sake of clarity we now illustrate how this problem can be written as a generic assignment problem.

Let us introduce $|\mathcal{N}|-|\mathcal{F}|$ fictitious channels. Let us define a modified cost function $C_{i j}=J_{\{i\}, k}, \forall j \in F$ and $C_{i j}=0$ otherwise. We adopt $\sum_{j} r_{i, j, k}$ as the decision variables. We note that since the channels are reliable, we have $\sum_{j} r_{i, j, k}=$ $\delta_{i, k}$. Let $\overline{\mathcal{N}}=\mathcal{N} \times \mathcal{N}$. Now (13) can be equivalently written as

$$
\max _{r_{i, j, k} \in\{0,1\}} \sum_{(i, j) \in \overline{\mathcal{N}}} C_{i j} r_{i, j, k}
$$

subject to (8) and (9). We recognize this as the form of a generic assignment problem. Assignment problems can be solved efficiently by the Hungarian method or auction-based algorithms [19], [20]. We note that the above symmetric form of an assignment problem with equal number of channels and agents is introduced for the sake of ease of exposition and the asymmetric form with smaller number of channels can be also efficiently solved by a variant of standard auction algorithms [20].

2) Unreliable Channels: Let $S_{c}$ denote the set of indices of the agents who make a communication attempt, i.e., $S_{c}=\left\{i: \sum_{j} r_{i, j, k}=1\right\}$. We note that we may have $S_{c}=\mathcal{N}$ depending on the channel availability. Let $\bar{j}(i)$ : $S_{c} \rightarrow\{1, \ldots, r\}$ denote the index $j$ with $r_{i, j, k}=1$ for $i \in S_{c}$. Let $\bar{j}_{i}$ denote $\bar{j}(i)$ for notational convenience. We observe that

$$
\begin{aligned}
\mathbb{E}\left\{J_{k} \mid \mathcal{R}\right\} & =\sum_{i \in \bar{S}_{c}} E_{i, k}^{0}+\sum_{i \in S_{c}}\left(E_{i, k}^{0}\left(1-q_{i \bar{j}_{i}}\right)+E_{i, k}^{1} q_{i \bar{j}_{i}}\right) \\
& =\sum_{i \in \mathcal{N}} E_{i, k}^{0}+\sum_{i \in S_{c}}\left(E_{i, k}^{1}-E_{i, k}^{0}\right) q_{i \bar{j}_{i}} \\
& =\sum_{i \in \mathcal{N}} E_{i, k}^{0}-\sum_{i \in S_{c}}\left(J_{\{i\}, k}\right) q_{i \bar{j}_{i}}
\end{aligned}
$$

Since the first term does not depend on $r_{i, j, k}$, minimizing $\mathbb{E}\left\{J_{k} \mid \mathcal{R}\right\}$ is equivalent to the following problem:

$$
\max _{r_{i, j, k} \in\{0,1\}} \sum_{i \in S_{c}} J_{\{i\}, k} q_{i \bar{j}_{i}}
$$

This expression can be informally interpreted as the scheme that pairs the agents with the highest cost $J_{\{i\}, k}$ with the channels that provide them with the highest probability of success $q_{i \bar{j}_{i}}$. Similar to the reliable channel case, (28) can be also written as an assignment problem and solved efficiently by the Hungarian method or auction-based algorithms.

\section{B. Coupled Dynamics}

Here we consider a system where agents may have coupled dynamics and observations of states of other agents. The cost cannot be decomposed into individual costs, but given that the decision maker has all the information it can compute how the posterior probability changes for different combinations of transmissions. As identified in other works in the literature, the problem is $\mathcal{N} \mathcal{P}$-hard, and its complexity grows exponentially with the number of agents.

If some of the subsystems are coupled in both dynamics and cost function whereas some are not, then we can decouple the cost function based on the coupled clusters of subsystems. For instance, one can rewrite the resource allocation problem for the reliable channels scenario as

$$
J_{k}=\min _{\mathcal{S}:|\mathcal{S}| \leq r} J_{\overline{\mathcal{S}}, k}^{\mathrm{up}}=\min _{\mathcal{S}:|\mathcal{S}| \leq r} \mathbb{E}\left\{\sum_{\mathcal{C}_{j}} J_{\overline{\mathcal{S}}, k, \mathcal{C}_{j}}^{\mathrm{up}}\right\},
$$

where $\mathcal{C}_{j} \subseteq \mathcal{N}, \mathcal{C}_{j_{1}} \cap \mathcal{C}_{j_{2}}=\emptyset$ and $\cup_{\ell} \mathcal{C}_{j_{\ell}}=\mathcal{N}$. Here $J_{\overline{\mathcal{S}}, k, \mathcal{C}_{j}}^{\text {up }}$ is the cost associated with cluster $\mathcal{C}_{j}$.

In what follows, we will implement the framework described in this section for agents with linear dynamics and with a quadratic cost function. A numerical example in Section VII with LQG dynamics shows the CoIL for such clusters. 


\section{COIL FOR DECOUPLED LQG}

In this section, we consider a system in which agents have decoupled, linear dynamics and observations. The system dynamics are

$$
\begin{aligned}
x_{i, k+1} & =A_{i} x_{i, k}+B_{i} u_{i, k}+w_{i, k}, \\
y_{i, k} & =C_{i} x_{i, k}+v_{i, k},
\end{aligned}
$$

where $A_{i}, B_{i}$ and $C_{i}$ are matrices of appropriate dimensions.

We consider a quadratic cost for a finite horizon $M$, i.e.,

$$
J_{o}=\mathbb{E}\left\{x_{M}^{T} Q_{M} x_{M}+\sum_{k=0}^{M-1}\left(x_{k}^{T} Q x_{k}+u_{k}^{T} R u_{k}\right)\right\},
$$

where $Q_{M}=\operatorname{diag}\left(Q_{1, M}, \ldots, Q_{N, M}\right), \quad Q=$ $\operatorname{diag}\left(Q_{1}, \ldots, Q_{N}\right), \quad R=\operatorname{diag}\left(R_{1}, \ldots, R_{N}\right)$, and $Q_{i, M}, Q_{i}, R_{i} \succ 0$ are of appropriate dimensions. In this case, the only coupling between the agents is via the scarce resources available and, hence, allocation.

\section{A. Control}

The solution of the control problem $\min _{u_{0}, \ldots, u_{M-1}} J$ subject to (17) is given by the control strategy

$$
u_{i, k}=L_{i, k} \hat{x}_{i, k \mid k}
$$

where $L_{i, k}$ is the feedback matrix and it is given by

$$
L_{i, k}=-\left(B_{i}^{T} \Pi_{i, k+1} B_{i}+R_{i}\right)^{-1} B_{i}^{T} \Pi_{i, k+1} A_{i},
$$

and where the symmetric positive semidefinite matrix $\Pi_{i}$ satisfies the discrete-time algebraic Riccati equation (DARE)

$$
\Pi_{i, k}=A_{i}^{T} \Pi_{i, k+1} A_{i}+Q_{i}-L_{i, k}^{T}\left(B_{i}^{T} \Pi_{i, k+1} B_{i}+R_{i}\right) L_{i, k} .
$$

Remark 1: Note that the feedback gain $L_{i, k}$ depends only on the system dynamics $\left(A_{i}, B_{i}\right)$ and the parameters of the cost function $\left(Q_{i, M}, Q_{i}, R_{i}\right)$; it is, therefore, independent of the characteristics of the disturbances. In the infinite horizon case, $\Pi_{i, k}$ converges to $\Pi_{i}$ that satisfies (21) and therefore $L_{i, k}$ converges to $L_{i}$.

\section{B. Estimation}

Let the a posteriori and a priori state estimates denoted by $\hat{x}_{i, k \mid k}=\mathbb{E}\left\{x_{i, k} \mid \mathcal{Y}_{i, k}\right\}$ and $\hat{x}_{i, k+1 \mid k}=\mathbb{E}\left\{x_{i, k+1} \mid \mathcal{Y}_{i, k}\right\}$, respectively. Also, let the corresponding error covariances given by

$$
\begin{aligned}
P_{i, k \mid k} & =\mathbb{E}\left\{\left(x_{k}-\hat{x}_{i, k \mid k}\right)\left(x_{k}-\hat{x}_{i, k \mid k}\right)^{T} \mid \mathcal{Y}_{k}\right\}, \\
P_{i, k+1 \mid k} & =\mathbb{E}\left\{\left(x_{k}-\hat{x}_{i, k+1 \mid k}\right)\left(x_{k}-\hat{x}_{i, k+1 \mid k}\right)^{T} \mid \mathcal{Y}_{k}\right\} .
\end{aligned}
$$

Then, by standard results in linear estimation theory, the Kalman filter is the minimum mean square estimate (MMSE), provided the noises are assumed Gaussian; otherwise, it is the best linear unbiased estimate. Let $A_{L, i, k} \triangleq$
$A_{i}+B_{i} L_{i, k}$. Then, the Kalman filter equations, based on [21], mutatis mutandis are given by

$$
\begin{aligned}
\hat{x}_{i, k+1 \mid k} & =A_{i} \hat{x}_{i, k \mid k}+B_{i} u_{i, k}=A_{L, i, k} \hat{x}_{i, k \mid k}, \\
P_{i, k+1 \mid k} & =A_{L, i, k} P_{i, k \mid k} A_{L, i, k}^{T}+W_{i}, \\
K_{i, k+1} & =P_{i, k+1 \mid k} C_{i}^{T}\left(C_{i} P_{i, k+1 \mid k} C_{i}^{T}+V_{i}\right)^{-1}, \\
\hat{x}_{i, k+1 \mid k+1} & =\hat{x}_{i, k+1 \mid k}+\delta_{i, k+1} K_{i, k+1}\left(y_{i, k+1}-C_{i} \hat{x}_{i, k+1 \mid k}\right), \\
P_{i, k+1 \mid k+1} & =\left(I-\delta_{i, k+1} K_{i, k+1} C_{i}\right) P_{i, k+1 \mid k} .
\end{aligned}
$$

Remark 2: Certainty Equivalence principle states that the optimal solution of a linear quadratic optimal control problem subject to uncertainties is the same as for the corresponding deterministic problem as long as the disturbances present in the stochastic control system are zero mean [22]. For this problem setup, it is shown in [23] that this is equivalent to saying that as long as the scheduling decisions are not a function of the applied control actions $\mathcal{U}_{k-1} \triangleq$ $\left\{u_{0}, \ldots, u_{k-1}\right\}$ certainty equivalence holds. Since, our control strategy depends only on $\hat{x}_{k \mid k}$, Certainty Equivalence holds; see also [24, Theorem 3.8].

Remark 3: Let $e_{i, k} \triangleq x_{i, k}-\hat{x}_{i . k \mid k}$, then the dynamics of the closed loop system are given by

$$
\begin{aligned}
x_{i, k+1}= & \left(A_{i}+B_{i} L_{i, k}\right) x_{i, k}-B_{i} L_{i, k} e_{i, k}+w_{i, k} \\
e_{i, k+1}= & \left(I-\delta_{i, k+1} K_{i, k+1} C_{i}\right) A_{i} e_{i, k} \\
& \quad+\left(I-\delta_{i, k+1} K_{i, k+1} C_{i}\right) w_{i, k}-\delta_{i, k+1} K_{i, k+1} .
\end{aligned}
$$

The eigenvalues of the closed loop system are the eigenvalues of $\left(A_{i}+B_{i} L_{i, k}\right)$ and $\left(I-\delta_{i, k+1} K_{i, k+1} C_{i}\right) A$. In this work, we assume that the resource allocation is such that the closed loop system is stable.

\section{Resource Allocation}

Cost $J_{o}$ in (18) can be written as (see [25, Lemma 6.1, Chapter 8] or [14, Eq. (4)])

$$
\begin{aligned}
J_{o}= & \mathbb{E}\left\{x_{0}^{T} \Pi_{0} x_{0}\right\}+\mathbb{E}\left\{\sum_{k=0}^{M-1} w_{k}^{T} \Pi_{k+1} w_{k}\right\} \\
& +\mathbb{E}\left\{\sum_{k=0}^{M-1}\left(u_{k}+L_{k} x_{k}\right)^{T} \bar{\Gamma}_{k}\left(u_{k}+L_{k} x_{k}\right)\right\},
\end{aligned}
$$

where $\bar{\Gamma}_{k}=\left(B^{T} \Pi_{k+1} B+R\right)$. The first and second terms in (24) are independent of the control action or scheduling and, hence, we can omit them. Thus, we consider only the last term of (24), which after substituting (19), it becomes [14, Eq. (5)]

$$
J=\mathbb{E}\left\{\sum_{k=0}^{M-1}\left(x_{k}-\hat{x}_{k \mid k}\right)^{T} \Gamma_{k}\left(x_{k}-\hat{x}_{k \mid k}\right)\right\},
$$

where $\Gamma_{k}=L_{k}^{T}\left(B^{T} \Pi_{k+1} B+R\right) L_{k}$.

In this work, we are interested in deriving a scheduling policy for the infinite horizon case. Hence, by letting $M$ become very large, then due to the fact that the system is stable, $\Gamma_{k}$ converges, i.e., $\lim _{M \rightarrow \infty} \Gamma_{k} \rightarrow \Gamma$, where $\Gamma=$ 
$L^{T}\left(B^{T} \Pi B+R\right) L$. We note that since the dynamics are decoupled, $\Gamma=\operatorname{diag}\left(\Gamma_{i}\right)$ and

$$
\begin{aligned}
J_{k} & =\sum_{i \in \mathcal{N}} \mathbb{E}\left\{\left(x_{i, k}-\hat{x}_{i, k \mid k}\right)^{T} \Gamma_{i}\left(x_{i, k}-\hat{x}_{i, k \mid k}\right)\right\} \\
& =\sum_{i \in \mathcal{N}} \mathbb{E}\left\{e_{i, k}^{T} \Gamma_{i} e_{i, k}\right\} \\
& =\sum_{i \in \mathcal{N}} \operatorname{tr}\left(\Gamma_{i} \mathbb{E}\left\{e_{i, k} e_{i, k}^{T}\right\}\right) \\
& =\sum_{i \in \mathcal{S}} \operatorname{tr}\left(\Gamma_{i} P_{i, k \mid k-1}\right)+\sum_{i \in \overline{\mathcal{S}}} \operatorname{tr}\left(\Gamma_{i} P_{i, k \mid k}\right) .
\end{aligned}
$$

The CoIL for agent $i$ in this scenario is given by

$$
\operatorname{CoIL}_{i}=\operatorname{tr}\left(\Gamma_{i}\left(P_{i, k \mid k}-P_{i, k \mid k-1}\right)\right) .
$$

Hence, for the case of reliable communication links, the scheduling optimization problem becomes

$$
\bar{J}_{k}=\min _{\mathcal{S}} \sum_{i \in \mathcal{S}} \operatorname{tr}\left(\Gamma_{i}\left(P_{i, k \mid k}-P_{i, k \mid k-1}\right)\right),
$$

which corresponds to (12). In the case of unreliable communication links the optimization becomes

$$
\max _{r_{i, j, k} \in\{0,1\}} \sum_{i \in S_{c}} \operatorname{tr}\left(\Gamma_{i}\left(P_{i, k \mid k}-P_{i, k \mid k-1}\right)\right) q_{i \bar{j}_{i}},
$$

where the notation is as introduced before.

\section{COIL FOR COUPLED LQG}

In this section, we consider a system in which agents with linear dynamics may have coupled dynamics and observations of states of other agents. The system dynamics are given by

$$
\begin{aligned}
x_{i, k+1} & =A_{i} x_{i, k}+\sum_{j \in\left|\mathcal{N}_{i}^{\text {in }}\right|} A_{i j} x_{j, k}+B_{i} u_{i, k}+w_{i, k}, \\
y_{i, k} & =C_{i} x_{k}+v_{i, k}
\end{aligned}
$$

where $A_{i j}$ is a matrix of appropriate dimensions.

We consider the same quadratic cost function as in (18), with the difference that now $Q_{M}, Q, R \succ 0$ are not necessarily block diagonal as before.

\section{A. Control}

As it is stated in Remark 1, the feedback gain $L_{k}$ will be independent of the statistics of the observations and it will converge to a matrix $L$ that it will no longer be block diagonal. Hence, the controller of agent $i$ will be of the form

$$
u_{i, k}=[L]_{i, \bullet} \hat{x}_{k \mid k},
$$

where $[L]_{i, \bullet}$ denotes the rows of $L$ corresponding to agent $i$ and all the columns. As a result, the decentralized structure of the system can be maintained, since both $[L]_{i, \bullet}$ and $\hat{x}_{k \mid k}$ are computed centrally and $u_{i, k}$ is communicated reliably over the downlink channel.

\section{B. Estimation}

Since only a subset of measurements reach the estimator, we define the block diagonal matrix $\Delta_{k} \in \mathbb{R}^{n_{y}}$, such that block $\left[\Delta_{k}\right]_{i}$ is given by

$$
\left[\Delta_{k}\right]_{i}= \begin{cases}I_{n_{y_{i}}}, & \text { if } \delta_{i, k}=1, \\ 0_{n_{y_{i}}}, & \text { otherwise, }\end{cases}
$$

where $I_{n_{y_{i}}}$ and $0_{n_{y_{i}}}$ are the identity and zero matrices of dimensions $n_{y_{i}} \times n_{y_{i}}$, respectively. The measurement equation (29b) for the whole system, now becomes

$$
y_{k}=\Delta_{k} C x_{k}+\eta_{k},
$$

where $C=\left[\begin{array}{lll}C_{1} & \ldots C_{|\mathcal{N}|}\end{array}\right]^{T}$ and $\eta_{k}=\Delta_{k} v_{k}$ is a stochastic disturbance with zero mean and finite second order matrix $H_{k}$. Let $A_{L} \triangleq A+B L$ and $C_{k} \triangleq \Delta_{k} C$. Then, the Kalman filter equations become

$$
\begin{aligned}
\hat{x}_{k+1 \mid k} & =A \hat{x}_{k \mid k}+B u_{k}=A_{L} \hat{x}_{k \mid k}, \\
P_{k+1 \mid k} & =A_{L} P_{k \mid k} A_{L}^{T}+W, \\
K_{k+1} & =P_{k+1 \mid k} C_{k+1}^{T}\left(C_{k+1} P_{k+1 \mid k} C_{k+1}^{T}+H_{k}\right)^{-1}, \\
\hat{x}_{k+1 \mid k+1} & =\hat{x}_{k+1 \mid k}+K_{k+1}\left(y_{k+1}-C_{k+1} \hat{x}_{k+1 \mid k}\right), \\
P_{k+1 \mid k+1} & =\left(I-K_{k+1} C_{k+1}\right) P_{k+1 \mid k} .
\end{aligned}
$$

Remark 4: Let $e_{k} \triangleq x_{k}-\hat{x}_{k \mid k}$, then the dynamics of the closed loop system are given by

$$
\begin{aligned}
x_{k+1}= & A_{L} x_{k}-B L_{k} e_{k}+w_{k} \\
e_{k+1}= & \left(I-K_{k+1} C_{k+1}\right) A e_{k} \\
& \quad+\left(I-K_{k+1} C_{k+1}\right) w_{k}-\delta_{k+1} K_{k+1} .
\end{aligned}
$$

The eigenvalues of the closed loop system are the eigenvalues of $A_{L}$ and $\left(I-K_{k+1} C_{k+1}\right) A$.

\section{Resource Allocation}

In the general case, we can consider the optimization

$$
\begin{aligned}
\bar{J}_{k} & =\min _{\delta_{k}: \sum_{i} \delta_{i, k} \leq r} \mathbb{E}\left\{e_{k}^{T} \Gamma e_{k}\right\} \\
& =\min _{\delta_{k}: \sum_{i} \delta_{i, k} \leq r} \operatorname{tr}\left(\Gamma \mathbb{E}\left\{e_{k} e_{k}^{T}\right\}\right) \\
& =\min _{\delta_{k}: \sum_{i} \delta_{i, k} \leq r} \operatorname{tr}\left(\Gamma P_{k \mid k}\right) .
\end{aligned}
$$

where $e_{k}=x_{k}-\hat{x}_{k \mid k}$. The cost cannot be decomposed into individual costs, but given that the decision maker has all the information it can compute how $P_{k \mid k}$ changes for different combinations of transmissions. As identified in other works in the literature, the problem is $\mathcal{N} \mathcal{P}$-hard, and its complexity grows exponentially with the number of agents; approximation methods can be used, but it is out of the scope of this paper.

\section{ILlustrative EXAMPLES}

To demonstrate how the system behaves under limited communication resources, we consider a toy example of a system consisting of 3 agents and at each time step, only 2 of them can communicate with the central estimator. Using this setup, we investigate different scenarios. As a cost function, 
we consider a quadratic cost with $Q=I$, where $I$ is the identity matrix of appropriate dimensions, and $R=0.01 I$. The process and measurement noises are given by

$$
W_{i}=\left[\begin{array}{cc}
0.03 & 0 \\
0 & 0.01
\end{array}\right] \text { and } V_{i}=\left[\begin{array}{cc}
0.01 & 0 \\
0 & 0.05
\end{array}\right] \text {, }
$$

respectively.

\section{A. Nonidentical agents with decoupled dynamics}

In this scenario, we consider 3 agents with different dynamics. At every time step 2 out of 3 agents can transmit their measurement to the estimator. In Fig. 2, we see the performance of the system. Specifically, in the top figure we compute the expected cost for each agent pair selection at each step and the one giving the minimum cost is chosen for transmission. The bottom figure shows how the system switches between different modes of operation $(\ell \in\{1,2,3\}$ corresponds to the case agent $\ell$ does not transmit its measurement). We observe that it is necessary to give more resources to some of the agents.
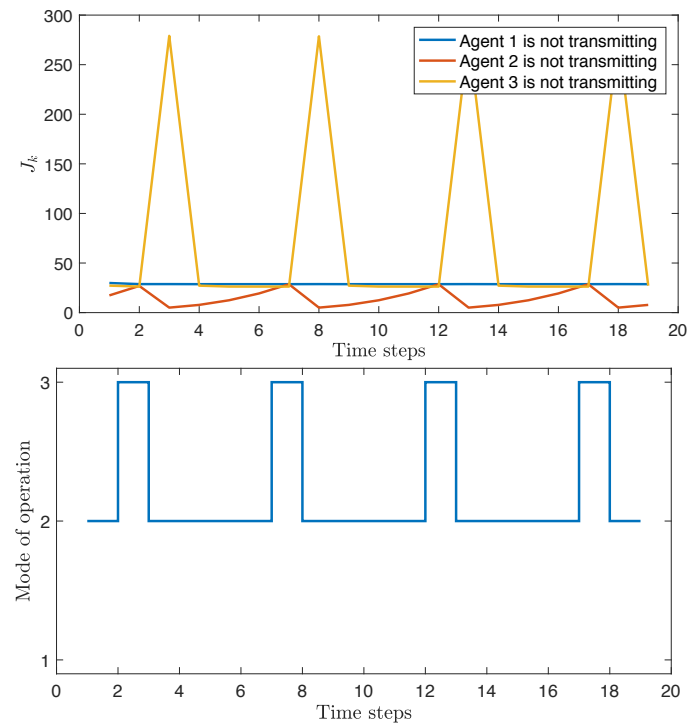

Fig. 2. Scheduling for a system with decoupled nonidentical agents. In the top figure the expected cost for each agent pair selection at each step is shown, justifying that the some agents need more resources than others (bottom figure).

Note that a round robin approach would have increased the cost of the system. We compare the cost at each time step for our method and round robin and the cost for each approach at each time step is shown in Fig. 3. Our method had a total cost reduction by $29 \%$.

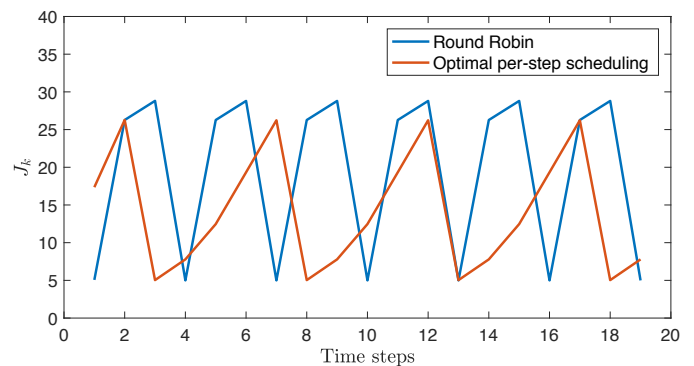

Fig. 3. Cost per time step for our method (red) and round robin approach (blue).

\section{B. Part of agents with decoupled dynamics}

In this scenario, we consider 3 agents, 2 of which are coupled. More specifically, the coupled dynamics of agents 1 and 2 are given by

$$
\begin{aligned}
A_{1,2} & =\left[\begin{array}{cccc}
1 & 0.1 & 0.2 & -0.1 \\
0 & 1 & 0 & 0.4 \\
0.2 & -0.1 & 1 & 0.1 \\
0 & 0.4 & 0 & 1
\end{array}\right], \\
B_{1,2} & =\left[\begin{array}{cccc}
0.1 & 0.5 & 0 & 0 \\
0.05 & 0.5 & 0 & 0 \\
0 & 0 & 0.1 & 0.5 \\
0 & 0 & 0.05 & 0.5
\end{array}\right] .
\end{aligned}
$$

The dynamics of agent 3 are

$$
A_{3}=\left[\begin{array}{cc}
1 & 0.1 \\
0 & 1
\end{array}\right], B_{3}=\left[\begin{array}{cc}
0.1 & 0.5 \\
0.05 & 0.5
\end{array}\right],
$$

and $C_{i}=I$ for all 3 agents.
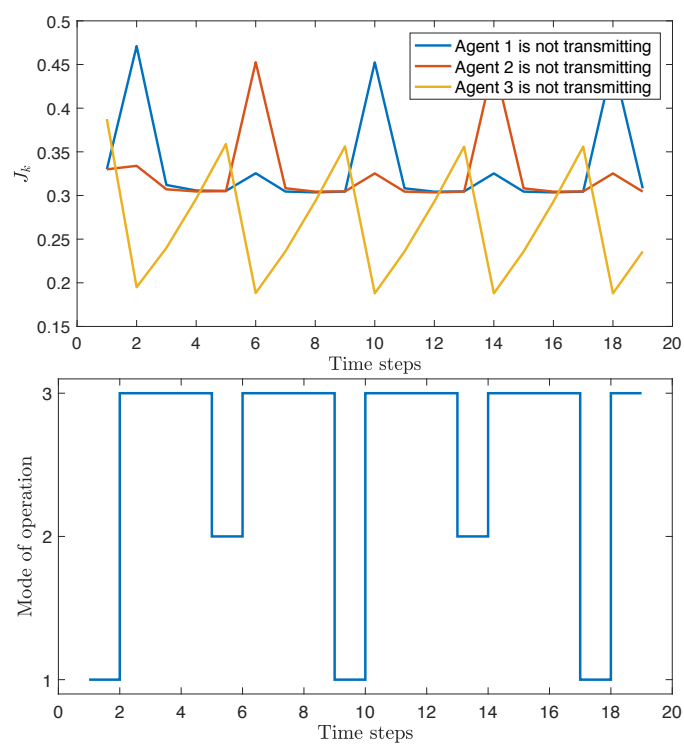

Fig. 4. Scheduling for a system in which some of the agents are coupled.

Fig. 4 shows that for the given dynamics more resources are needed to be allocated to the coupled systems, whereas if we run another example in which the dynamics of agent 3 are changed to

$$
A_{3}=\left[\begin{array}{cc}
1.2 & 0.1 \\
0 & 1.1
\end{array}\right]
$$

we observe that agent 3 needs more resources and the coupled system is "sampled" alternatively; this is demonstrated in Fig. 5.

\section{CONCLUSIONS AND Future DiRECTIONS}

\section{A. Conclusions}

In this paper, we considered the scheduling problem of a decentralized system where a number of dynamical subsystems with no computational power are scheduled to transmit their measurements via a resource-limited communication 



Fig. 5. Scheduling for a system in which some of the agents are coupled.

network to a remote decision maker who acts as an estimator, controller and scheduler for the subsystems. We proposed a new approach for communication resource allocation for a wide class of objective functions, for both coupled and decoupled systems, and for both scheduling observations as well as control commands. The proposed framework allows to schedule over a finite horizon and can explicitly deal with stochastic channels. For decoupled subsystems, we proposed the notion of CoIL and we demonstrated that the communications resource allocation problem can be directly expressed in terms of CoIL functions as an assignment-type optimization problem. Illustrative examples demonstrated how communication resources affect the performance of the system.

\section{B. Future Directions}

It was proven in [9] that any schedule that has a bounded average estimation error can be arbitrarily approximated by bounded periodic schedules. We wish to develop a systematic way of designing periodic schedules for these cases. Part of ongoing research considers the case for which the uplink channels that are non-orthogonal to each, thus having agents experiencing interference. In addition, we plan to consider the case for which the downlink communication channels can be in outage as well. Finally, an interesting yet challenging scenario will be to consider networks of control systems that have a graph like structure in both interactions and communication links, and design distributed scheduling algorithms.

\section{REFERENCES}

[1] W. Zhang, M. S. Branicky, and S. M. Phillips, "Stability of networked control systems," IEEE Control Systems, vol. 21, no. 1, pp. 84-99, Feb 2001.

[2] L. Schenato, B. Sinopoli, M. Franceschetti, K. Poolla, and S. S. Sastry, "Foundations of Control and Estimation Over Lossy Networks," Proceedings of the IEEE, vol. 95, no. 1, pp. 163-187, Jan. 2007.
[3] O. C. Imer, S. Yüksel, and T. Başar, "Optimal control of LTI systems over unreliable communication links," Automatica, vol. 42, no. 9, pp. 1429-1439, Sep. 2006.

[4] V. Gupta, T. H. Chung, B. Hassibi, and R. M. Murray, "On a stochastic sensor selection algorithm with applications in sensor scheduling and sensor coverage," Automatica, vol. 42, no. 2, pp. 251-260, 2006.

[5] Y. Mo, R. Ambrosino, and B. Sinopoli, "Sensor selection strategies for state estimation in energy constrained wireless sensor networks," Automatica, vol. 47, no. 7, pp. 1330-1338, Jul. 2011.

[6] C. Yang, J. Wu, X. Ren, W. Yang, H. Shi, and L. Shi, "Deterministic Sensor Selection for Centralized State Estimation Under Limited Communication Resource," IEEE Transactions on Signal Processing, vol. 63, no. 9, pp. 2336-2348, May 2015.

[7] A. S. Leong, S. Dey, and D. E. Quevedo, "Sensor Scheduling in Variance Based Event Triggered Estimation with Packet Drops," IEEE Transactions on Automatic Control, 2016.

[8] L. Shi, P. Cheng, and J. Chen, "Optimal Periodic Sensor Scheduling With Limited Resources," IEEE Transactions on Automatic Control, vol. 56, no. 9, pp. 2190-2195, Sept 2011.

[9] Y. Mo, E. Garone, and B. Sinopoli, "On infinite-horizon sensor scheduling," Systems \& Control Letters, vol. 67, pp. 65-70, 2014.

[10] L. Zhao, W. Zhang, J. Hu, A. Abate, and C. J. Tomlin, "On the Optimal Solutions of the Infinite-Horizon Linear Sensor Scheduling Problem," IEEE Transactions on Automatic Control, vol. 59, no. 10, pp. 28252830, Oct. 2014.

[11] D. Han, J. Wu, H. Zhang, and L. Shi, "Optimal sensor scheduling for multiple linear dynamical systems," Automatica, vol. 75, pp. 260-270, Jan. 2017.

[12] C. Ramesh, H. Sandberg, and K. H. Johansson, "Design of State-Based Schedulers for a Network of Control Loops," IEEE Transactions on Automatic Control, vol. 58, no. 8, pp. 1962-1975, Aug 2013.

[13] X. Shen and P. K. Varshney, "Sensor Selection Based on Generalized Information Gain for Target Tracking in Large Sensor Networks," IEEE Transactions on Signal Processing, vol. 62, no. 2, pp. 363-375, Jan. 2014.

[14] A. Molin, C. Ramesh, H. Esen, and K. H. Johansson, "Innovationsbased priority assignment for control over CAN-like networks," in IEEE Conference on Decision and Control (CDC), Dec 2015, pp. 4163-4169.

[15] F. Zhao, J. Shin, and J. Reich, "Information-driven dynamic sensor collaboration," IEEE Signal processing magazine, vol. 19, no. 2, pp. $61-72,2002$.

[16] D. Han, J. Wu, Y. Mo, and L. Xie, "Stochastic sensor scheduling for multiple dynamical processes over a shared channel," in IEEE Conference on Decision and Control (CDC), 2016, pp. 6315-6320.

[17] Yongmian Zhang and Qiang Ji, "Efficient Sensor Selection for Active Information Fusion," IEEE Transactions on Systems, Man, and Cybernetics, Part B (Cybernetics), vol. 40, no. 3, pp. 719-728, Jun. 2010

[18] T. Soleymani, S. Hirche, and J. S. Baras, "Optimal Information Control in Cyber-Physical Systems," IFAC-PapersOnLine, vol. 49, no. 22, pp. 1-6, 2016.

[19] D. P. Bertsekas and D. A. Castañon, "Parallel synchronous and asynchronous implementations of the auction algorithm," Parallel Computing, vol. 17, no. 6-7, pp. 707-732, 1991.

[20] D. P. Bertsekas, "Auction algorithms for network flow problems: A tutorial introduction," Computational optimization and applications, vol. 1, pp. 7-66, 1992.

[21] B. Sinopoli, L. Schenato, M. Franceschetti, K. Poolla, M. I. Jordan, and S. S. Sastry, "Kalman filtering with intermittent observations," IEEE Transactions on Automatic Control, vol. 49, no. 9, pp. 14531464, Sept 2004.

[22] Y. Bar-Shalom and E. Tse, "Dual effect, certainty equivalence, and separation in stochastic control," IEEE Transactions on Automatic Control, vol. 19, no. 5, pp. 494-500, Oct 1974.

[23] C. Ramesh, H. Sandberg, L. Bao, and K. H. Johansson, "On the dual effect in state-based scheduling of networked control systems," in Proceedings of the 2011 American Control Conference, June 2011, pp. 2216-2221.

[24] C. Ramesh, "State-based channel access for a network of control systems," Ph.D. dissertation, KTH Royal Institute of Technology, 2014.

[25] K. J. Åström, Introduction to stochastic control theory, ser. Mathematics in science and engineering. New York, London: Academic press, 1970, vol. 70 . 